\title{
Timing conditions and the magnitude of gender differences on the Mental Rotations Test
}

\author{
DANIEL VOYER \\ University of New Brunswick, Fredericton, New Brunswick, Canada \\ MARGUERITE A. RODGERS \\ University of British Columbia, Vancouver, British Columbia, Canada \\ and \\ PETER A. MCCORMICK \\ St. Francis Xavier University, Antigonish, Nova Scotia, Canada
}

\begin{abstract}
In two experiments, we examined the effect of timing conditions on the magnitude of gender differences in performance on the Mental Rotations Test (MRT). In Experiment 1, each of 196 females and 119 males was administered the MRT via a Microsoft PowerPoint presentation in one of five timing conditions $(15,20,25,30$, and $40 \mathrm{sec})$. The participants were exposed to each MRT item for the period specified in the assigned timing condition. Experiment 2 was conducted to address flaws found in Experiment 1 . Accordingly, each of 105 females and 105 males was individually administered the task in one of three timing conditions ( $15 \mathrm{sec}, 25 \mathrm{sec}$, or unlimited duration). The results of both experiments showed that the magnitude of gender differences was similar across timing conditions when a conventional scoring method was used. An analysis of guessing behavior generally indicated that men tend to show little effect of timing conditions, whereas women's propensity to guess increases when they are given more time to respond. In general, the results supported an interpretation of gender differences on the MRT that relies on the joint operation of performance factors and level of spatial ability.
\end{abstract}

Individual differences in spatial abilities are commonly observed in everyday life-for example, in the fact that some people are able to read maps better than others. Such individual differences presumably originate from a variety of sources. Gender is one individual difference variable commonly claimed to be related to variations in spatial abilities. Indeed, the existence of gender differences in spatial abilities favoring males has been well established in the psychological literature (Linn \& Petersen, 1985; Voyer, Voyer, \& Bryden, 1995), despite some controversy regarding the nature and definition of those abilities (Caplan, MacPherson, \& Tobin, 1985).

Before we discuss gender differences in spatial abilities, it is necessary to define clearly what such abilities are. According to Caplan et al. (1985), the lack of consensus in defining spatial abilities is a serious obstacle in the investigation of gender differences. Linn and Petersen (1985) suggested that most investigators agree that spatial ability involves multiple processes. From that perspective, they

The study reported in this article was supported by grants from the Natural Science and Engineering Research Council of Canada to D.V. and P.A.M. The authors thank Rebecca Brewer, Tanya Poitras, Amy Terry, and Marie-Eve Vallée for their assistance with data collection and scoring. Correspondence concerning this article may be addressed to D. Voyer, Department of Psychology, University of New Brunswick, Bag Service \#45444, Fredericton, New Brunswick E3B 6E4, Canada (email:voyer@unb.ca). described three categories of spatial abilities, which they labeled spatial perception, mental rotation, and spatial visualization. In tests measuring spatial perception, participants determine spatial relations with respect to the orientation of their own bodies or in the context of distracting information. Mental rotation tasks are used to examine the ability to rotate a 2-D or 3-D figure rapidly and accurately in one's imagination. Tasks of spatial visualization involve complicated, multistep manipulations of spatially presented information. These tasks are distinguished from spatial perception and mental rotation tasks in that they include the possibility of multiple solution strategies. According to Linn and Petersen's meta-analysis, gender differences in spatial ability are large for mental rotation, medium for spatial perception, and virtually nonexistent for spatial visualization, with males generally showing the advantage.

The test that most consistently demonstrates gender differences in spatial abilities is the 3-D Mental Rotations Test (MRT) developed by Vandenberg and Kuse (1978). This test contains 20 items for each of which there are always two correct and two incorrect answers. The test uses depictions of 3-D cube figures designed by Shepard and Metzler (1971). According to Linn and Petersen (1985) and Voyer et al. (1995), the male advantage in spatial abilities is largest on Vandenberg and Kuse's MRT.

Many social and biological factors have been proposed to account for the prevalence of gender differences in 
favor of men in spatial abilities. Factors such as choice of activities and training (Baenninger \& Newcombe, 1989), gender role identification (Signorella \& Jamison, 1986), and problem-solving strategies (Allen \& Hogeland, 1978) are a few examples of social variables. Rate of maturation (Sanders \& Soares, 1986; Waber, 1976), genetic complement (McGee, 1979), and lateralization of function (Levy, 1971) are examples of biological variables.

In the present study, we focused on other possible variables: performance factors and work habits. The claim that these factors may account for gender differences on the MRT is based on Goldstein, Haldane, and Mitchell's (1990) observation that males tend to perform tasks quickly, whereas females tend to work more slowly and carefully. These authors had males and females perform the MRT with a 6-min time limit. The participants then drew a line after the last item they had attempted and completed the remaining items without time limits. Goldstein et al. predicted that, without time constraints, females would perform as well as males on tests of spatial ability. They found that when time limits were removed, the performance of females was no longer significantly poorer than that of males. Voyer (1997) used a between-subjects approach (as opposed to the within-subjects methodology used by Goldstein et al.) in which separate groups of participants completed the MRT either with or without time limits. His data supported Goldstein et al.'s interpretation, and he too suggested gender differences in procedural factors to explain gender differences on the MRT.

In contrast, Lohman (1986) argued that gender differences arise from differences in level of spatial ability and that the influence of procedural factors is minimal. He claimed that even when given unlimited time to perform mental rotation problems, as a group, females never reach the level of accuracy achieved by males. Although Lohman based his work on a careful examination of speedaccuracy tradeoff functions in a conventional mental rotation task in which a pair of figures was presented and a same-different judgment was made, his conclusion can be extended to the MRT. In this view, the removal of time constraints should not reduce the magnitude of gender differences on the MRT. Studies by Resnick (1993) and Masters (1998) support Lohman's claims. These authors both found that males performed significantly better on the MRT even without time constraints. Resnick administered the MRT only in an untimed condition, so comparison of the magnitude of gender differences with time limits is not possible and allows the argument that the magnitude of these differences might still be reduced significantly without time constraints. In contrast, Masters used a wellcontrolled study to examine the hypothesis that procedural factors affect the magnitude of gender differences on the MRT. According to her, the published studies, such as those by Goldstein et al. (1990), do not support the hypothesis that the gender difference on MRT is due to performance factors. She criticized Goldstein et al.'s methodology and implemented ways to improve it in her study. Specifically, she used a between-subjects design in which separate groups of participants completed the MRT with or without time limits. In addition, she used participant samples of sufficient size to discount possible Type II errors as an alternative explanation of her finding. The results of that study showed that unlimited time did not eliminate gender differences on the MRT. Thus, the two contradictory hypotheses are supported in the literature by different sets of data.

Studies of timing conditions on the MRT generally use only two timing conditions: timed and untimed (Goldstein et al., 1990). An untimed condition does not necessarily mean the absence of time pressure. The presence of other test takers, for example, is a factor that would produce some time pressure. Time pressure is likely to occur when faster participants complete the task and start fidgeting while they wait for others to finish. In contrast, the present study systematically manipulated time pressure by offering varied timing conditions. All participants who were tested at the same time were subjected to the same timing conditions, thus reducing pressure from fellow test takers.

The purpose of the present study was to examine the effect of timing conditions on the magnitude of gender differences on the MRT. This was achieved by presenting the MRT items one at a time for a fixed exposure duration that varied across conditions. It was expected that, if the approaches participants use to complete the MRT affects gender differences on that task, as proposed by Goldstein et al. (1990), those differences should be more pronounced with stricter time constraints, with males having the advantage, but they should decrease in magnitude as time constraints are relaxed. In statistical terms, the hypothesis based on Goldstein et al.'s rationale predicts a gender $\times$ timing condition interaction. However, if the level-ofspatial-ability hypothesis (Lohman, 1986) is correct, the magnitude of gender differences will remain the same across timing conditions. Statistically, the hypothesis based on Lohman's claim predicts only a main effect of gender, independent of timing conditions.

Voyer (1997) proposed an alternate way to examine scores on the MRT on the basis of the six possible outcomes of each test item. Specifically, on any given item a participant can have two correct choices (CC), one correct choice and one wrong choice $(\mathrm{CW})$, one correct choice and one blank (CB), two wrong choices (WW), one wrong choice and one blank (WB), or two blanks (BB). The stringent scoring procedure proposed by Vandenberg and Kuse (1978, pp. 599-600) and Vandenberg (1985) actually reflects the CC items. Therefore, the predictions presented above apply to CC items.

Given that the goal of the present study is to examine performance when a limited amount of time is allotted for each item, the outcomes presented by Voyer (1997) take on a different meaning. Specifically, in a conventional administration of the MRT, BB items found at the end of the test would represent items that were not completed in the allotted time and could be taken as a measure of completion speed. However, in the present study, BB items could reflect either performance factors under short exposure 
duration (not enough time to respond) or ability under longer durations (inability to reach a solution even with sufficient time). Thus, these items do not allow an examination of performance factors.

Voyer (1997) also argued that CW, CB, WB, and WW items should be considered as representing measures of guessing behavior on the MRT. According to Voyer, a large number of any of these items reflects a high propensity to guess. However, this reasoning can be viewed as incorrect on two points. First, items containing a blank (CB, WB) are ambiguous because they can be considered as items for which the participants knew (or thought they knew) one answer and refused to guess on the other. This is especially important when one considers that instructions for the MRT typically state that there are two responses for each item and discourage guessing. This means that, when producing an item with a blank, one likely ignores the aspect of the instructions concerning the two responses per item. From this perspective, any item with at least one blank (including BB items) can be considered as reflecting reluctance to guess, especially when sufficient time is given. These items are thus useful in examining guessing behavior, but a large number of such items should be interpreted as reflecting resistance to guessing or low propensity to guess. In contrast, CW and WW items have the highest likelihood of directly reflecting a certain amount of guessing, because it is plausible to believe that when both answers are attempted, a wrong response (W) may represent a case in which a guess was made. Thus, working on the assumption that $\mathrm{CW}$ and WW items provide the most direct measure of guessing, a large number of such items would indicate a high propensity to guess.

There is an obstacle to a clear interpretation of all the non-CC items. Because men are expected to obtain more $\mathrm{CC}$ items than women, they should produce a smaller overall number of non-CC items. Thus, if non-CC items were equally distributed among the remaining items, men would always have fewer CW, CB, WB, BB, and WW items than women would. One way to handle this problem is to use the ratios of $\mathrm{CW}, \mathrm{CB}, \mathrm{WB}, \mathrm{BB}$, and $\mathrm{WW}$ items over the total number of non-CC items as a dependent variable in an examination of guessing behavior. This would reflect the percentage of a given item in relation to the overall number of non-CC items. When this approach is used, according to Goldstein et al.'s (1990) hypothesis, women should get a smaller proportion of $\mathrm{CW}$ and $\mathrm{WW}$ items and more $\mathrm{CB}, \mathrm{WB}$, and $\mathrm{BB}$ items than men should, because they are less likely to guess than are men. However, a level-of-ability hypothesis would likely predict that females should get proportionally more $\mathrm{CW}$ and $\mathrm{WW}$ items and fewer CB, WB, and BB items than men because their limited level of spatial ability would compel them to guess.

In addition to the novel approach to the examination of guessing behavior proposed above, it is important to emphasize other original aspects of the present study. Specifically, in previous work with the MRT, a test booklet in which all the items are presented was used. This approach has the disadvantage that participants can skip the more difficult items in the hope of returning to them if time permits. Thus, the score on this test might be biased because low-ability participants might choose to answer only those questions that they deem easy. Considering that the original MRT constructed by Vandenberg and Kuse (1978) contained 10 (of a total of 20) items in which the distractors differed from the targets in terms of the actual block configurations used as well as in rotation, it can plausibly be argued that such items would be easier to complete. Thus, it is likely that some participants would tend to complete more of those items than the more difficult ones, in which distractors are a mirror image of the target. Unfortunately, in no published work has the differential performance been examined closely as a function of item type. In the present study, we dealt with this problem in two ways. First, only about one third of the items have distractors that differ in structure (see the Materials section below). Second, the use of an item-by-item presentation format increases the likelihood that participants will provide answers to all items (within the limits set by the time constraints). This is likely to provide a better measure of the participants' abilities in the task.

To summarize, in the present study we examined the opposing predictions made by Goldstein et al.'s (1990) performance factors hypothesis and Lohman's (1986) level-of-spatial-abilities hypothesis. These predictions were tested through an examination of performance on $\mathrm{CC}$ items as a function of timing conditions, reflecting performance factors, and of the relative proportions of $\mathrm{CW}, \mathrm{WW}, \mathrm{CB}, \mathrm{WB}$, and $\mathrm{BB}$ items, reflecting guessing behavior.

\section{EXPERIMENT 1}

\section{Method}

Participants. The participants were 315 (196 female, 119 male) undergraduate students enrolled in an introductory psychology course at Saint Francis Xavier University. The participants had normal or corrected-to-normal vision. Their participation was voluntary and was rewarded with course credit.

The participants were tested in six groups of approximately 55 persons each. Testing was conducted during regularly scheduled classes. The participants were told that they could withdraw from the experiment at any time without prejudice to their grade in the course, in agreement with the American Psychological Association ethical guidelines (APA, 1992) and the ethical requirements in place at St. Francis Xavier University. None of the participants chose to withdraw.

Materials. The MRT (Vandenberg \& Kuse, 1978) was used as a measure of spatial abilities. The version of the MRT used here is based on the redrawn test produced by Peters et al. (1995). It consists of 24 multiple-choice items each consisting of a target figure, two correct alternatives, and two distractors. The correct alternatives are always identical to the target but are shown in various orientations. For 15 of the items, the distractors were rotated mirror images of the target, whereas for the remaining items the distractors were rotated images of one of the other targets. The test consisted of three practice items followed by the 24 test items. Scores were calculated by computing for each participant the number of each of the six possible outcomes (CC, WW, CW, CB, WB, and BB) on MRT items pro- 
posed by Voyer (1997). The WW, CW, CB, WB, and BB items were divided by the total number of non-CC items to reflect percentages. It is important to remember that the $\mathrm{CC}$ items represent the scoring procedure recommended by Vandenberg and Kuse (1978) and Vandenberg (1985) and result in the maximum score of 24 . This procedure has been found to maximize the magnitude of gender differences on the MRT (Voyer et al., 1995).

The stimuli were projected by means of a Sharp xg-NV2U Notevision projector on a $2 \times 3 \mathrm{~m}$ white projection screen in an $8 \times 10 \mathrm{~m}$ room. Each of the five block configurations used on individual items was, on average, $19 \mathrm{~cm}$ wide and $19 \mathrm{~cm}$ high and was enclosed within a rectangular frame $26 \mathrm{~cm}$ high and $23 \mathrm{~cm}$ wide. The distance between the target stimulus and the first stimulus in each set of alternatives was $14 \mathrm{~cm}$, and a distance of $5 \mathrm{~cm}$ separated the alternatives.

Procedure. The test was administered one item at a time using Microsoft PowerPoint. Each item remained visible for the period specified in the assigned timing condition. For example, in the 15sec condition, each of the 3 practice items and 24 test items was presented for $15 \mathrm{sec}$. A slide on which the message "Get Ready" was centered appeared for $1 \mathrm{sec}$ before each item. In addition, a $500-\mathrm{Hz}$, 100 -msec tone with an approximate intensity of $80 \mathrm{~dB}$ (coming from the front of the testing room) was presented whenever a change of slide occurred. For each item, the participants were required to indicate which two of the four test figures were identical to the target figure by circling the correct answers on the answer sheet provided. Presentation time reflected all the time allotted for response; therefore, the participants were instructed to stop responding immediately when they heard the tone indicating that the slide had changed to the "Get Ready" slide. The instructions discouraged them from guessing by stating that their score would be negatively affected if they guessed.

Five different timing conditions were used: 15, 20, 25, 30, and $40 \mathrm{sec}$ per item. The shortest value was selected on the basis of pilot testing. ${ }^{1}$ Thus, the initial duration was set at $15 \mathrm{sec}$, with the other values increasing by $5 \mathrm{sec}$ up to a value of $30 \mathrm{sec}$. The final value of $40 \mathrm{sec}$ was presumed to be sufficiently long to accommodate a response by most of the participants and to reflect a clearly relaxed time constraint. The value of $20 \mathrm{sec}$ included in this range approximates the limits set by Goldstein et al. (1990) and Masters (1998) that is, 6 min for a 20 -item test (18 sec per item) - and the 25 -sec limit is equal to the 10 -min limits for 24 items given by Voyer (1997).

Testing during class precluded random assignment of participants to each experimental condition. Each class was therefore assigned to one of the five timing conditions. The distribution of participants over the 15-, 20-, 25-, 30-, and 40-sec conditions was 46, 39, 31, 31, and 49 , respectively, for females and $30,22,23,22$, and 22 , respectively, for males.

\section{Results}

The data were analyzed separately for each of the approaches used to examine the hypotheses. Therefore, an analysis of variance (ANOVA) with gender and timing condition $(15,20,25,30$, and $40 \mathrm{sec})$ as the independent variables and the number of $\mathrm{CC}$ items as the dependent variable was computed to test the hypothesis concerning performance factors. A multivariate ANOVA with gender and timing condition as the independent variables and the proportions of $\mathrm{WW}, \mathrm{CW}, \mathrm{CB}, \mathrm{WB}$, and $\mathrm{BB}$ items as dependent variables was performed to test the hypothesis concerning guessing behavior. Note that all multivariate tests of significance were based on Pillai's trace, since it is deemed to be most resistant to assumption violations (Tabachnick \& Fidell, 1996). In addition, all power calculations used an alpha level of .05.

$\mathrm{CC}$ as dependent variable. Analysis of the $\mathrm{CC}$ items revealed a significant effect of gender $[F(1,305)=41.39$, $\left.M S_{\mathrm{e}}=22.84, p<.01\right]$. An examination of the means in Table 1 reveals that the male participants obtained significantly more $\mathrm{CC}$ items than the female participants did.

A significant effect of timing condition was also found $\left[F(4,305)=23.31, M S_{\mathrm{e}}=22.84, p<.01\right]$. The means relevant to this finding are also presented in Table 1. Multiple comparisons with the Neuman-Keuls technique at the .05 level of significance revealed that the 40 -sec condition produced a better performance than did each of the other conditions. In addition, the 30 -sec condition resulted in a better performance than the 25-, 20-, and 15-sec conditions. No other differences were significant.

The interaction between gender and timing condition failed to achieve statistical significance $[F(4,305)=0.27$, $M S_{\mathrm{e}}=22.84$, n.s., power $\left.=.11\right]$. Nevertheless, Table 1 presents mean scores as a function of gender and timing conditions to better illustrate the pattern of results.

Finally, as an additional aid in examining the influence of time constraints on performance, Table 1 presents the percentage of participants who attempted all items. In this

Table 1

Mean Number of CC Items on the Mental Rotations Test and Percentage of Participants Attempting All Items as a Function of Timing Condition (in Seconds) and Gender in Experiment 1

\begin{tabular}{|c|c|c|c|c|c|c|c|c|c|c|c|c|}
\hline \multirow[b]{3}{*}{ Group } & \multicolumn{10}{|c|}{ Timing Condition } & & \\
\hline & \multicolumn{2}{|c|}{15} & \multicolumn{2}{|c|}{20} & \multicolumn{2}{|c|}{25} & \multicolumn{2}{|c|}{30} & \multicolumn{2}{|c|}{40} & \multicolumn{2}{|c|}{ Overall } \\
\hline & $M$ & $S D$ & $M$ & $S D$ & $M$ & $S D$ & $M$ & $S D$ & $M$ & $S D$ & $M$ & $S D$ \\
\hline \multicolumn{13}{|c|}{ CC Items } \\
\hline Women & 5.2 & 3.4 & 6.8 & 5.4 & 8.3 & 4.7 & 9.8 & 4.9 & 12.6 & 4.9 & 8.6 & 5.3 \\
\hline Men & 9.2 & 5.2 & 10.7 & 5.4 & 10.7 & 4.4 & 13.7 & 4.7 & 16.5 & 5.0 & 12.0 & 5.6 \\
\hline \multicolumn{13}{|c|}{ Percentage of Participants Attempting All Items } \\
\hline Women & \multicolumn{2}{|c|}{23.9} & \multirow{2}{*}{\multicolumn{2}{|c|}{$\begin{array}{l}33.3 \\
63.6\end{array}$}} & \multirow{2}{*}{\multicolumn{2}{|c|}{$\begin{array}{l}48.4 \\
56.5\end{array}$}} & \multicolumn{2}{|c|}{83.9} & \multicolumn{2}{|c|}{77.6} & \multicolumn{2}{|c|}{53.4} \\
\hline Men & \multicolumn{2}{|c|}{66.7} & & & & & \multicolumn{2}{|c|}{72.7} & \multicolumn{2}{|c|}{95.5} & \multicolumn{2}{|c|}{71.0} \\
\hline
\end{tabular}

Note-CC, two correct responses. The percentage of participants attempting all items was computed within each gender. 
context, an item was considered to have been attempted when at least one answer was circled, regardless of its accuracy. Significantly more men than women attempted all items at 15 and $20 \sec \left[\chi^{2}(1)=13.74, p<.01\right.$ and $\chi^{2}(1)=$ $5.25, p<.05$, respectively], and the difference approached significance at $40 \mathrm{sec}\left[\chi^{2}(1)=3.47, p<.06\right]$. No gender differences in completion rate were observed in the remaining conditions (smallest $p \geq .25$ ).

Proportions of $W W, C W, C B, W B$, and $B B$ as dependent variables. Analysis of the proportion of $\mathrm{WW}$, $\mathrm{CW}, \mathrm{CB}, \mathrm{WB}$, and $\mathrm{BB}$ items revealed a marginally significant multivariate effect of gender $[F(4,302)=2.36, p<$ .054]. Univariate tests of significance indicated that the effect of gender was significant on $\mathrm{CW}[F(1,305)=5.60$, $\left.M S_{\mathrm{e}}=643.07, p<.05\right]$ and $\mathrm{BB}\left[F(1,305)=6.47, M S_{\mathrm{e}}=\right.$ $115.34, p<.05$ ] items, but not on WW, CB, and WB items (smallest $p>.079$, maximum power $=.42$ ). An examination of the relevant means presented in Table 2 indicates that the men produced a significantly greater proportion of CW items than did the women, whereas the reverse pattern was observed on BB items.

A significant multivariate effect of timing condition was also found $[F(16,1220)=4.88, p<.01]$. Univariate tests of significance indicated that the effect of timing condition was significant on all of the items except WW $[F(4,305)=$ $16.36, M S_{\mathrm{e}}=643.07, p<.01$ for $\mathrm{CW} ; F(4,305)=9.83$, $M S_{\mathrm{e}}=421.84, p<.01$ for CB; $F(4,305)=4.24, M S_{\mathrm{e}}=$ $68.05, p<.05$ for WB; $F(4,305)=7.76, M S_{\mathrm{e}}=115.34$, $p<.01$ for BB; $F(4,305)=1.71, M S_{\mathrm{e}}=109.94$, n.s., for WW]. The means relevant to these findings are presented in Table 2. Essentially, the significant main effect of condition reflected a positive correlation between time and the proportion of $\mathrm{CW}$ items $[r(315)=.43, p<.01]$ and a negative correlation between time and proportions of $\mathrm{CB}$, $\mathrm{WB}$, and BB items $[r s(315)=-.32,-.27$, and -.29 , respectively; all $p$ s $<.01]$.

Finally, a significant gender $\times$ timing condition interaction was observed in the multivariate test $[F(16,1220)=$ $2.43, p<.01]$. Univariate tests of significance indicated that this interaction was significant on the proportions of $\mathrm{CW}$ items $\left[F(4,305)=3.40, M S_{\mathrm{e}}=643.07, p<.05\right]$, WB items $\left[F(4,305)=2.93, M S_{\mathrm{e}}=68.05, p<.05\right]$, and BB items $\left[F(4,305)=4.36, M S_{\mathrm{e}}=114.34, p<.01\right]$, but not on those of WW and CB items (smallest $p>.26$, maximum power $=.41$ ). The means relevant to this interaction are presented in Table 2 . This interaction essentially reflected the fact that the men showed a smaller correlation than the women between time and the proportion of $\mathrm{CW}$ items [women: $r(196)=.50, p<.01 ;$ men: $r(119)=.31$, $p<.01$ ], WB items [women: $r(196)=-.37, p<.01$; men: $r(119)=-.04$, n.s.], and BB items [women: $r(196)=-.38, p<.01$; men: $r(119)=-.15$, n.s.]

\section{Discussion}

In the present experiment, we investigated hypotheses pertaining to the approaches used by women and men when completing the MRT. The performance factors hypothesis on this matter predicted a significant gender $\times$ timing condition interaction, reflecting large gender differences for $\mathrm{CC}$ items in conditions with stringent time constraints and reduced gender differences when time constraints were relaxed. In contrast, the level-of-spatialability approach predicted gender differences of equal magnitude across timing conditions for $\mathrm{CC}$ items. The results of this analysis supported the predictions on the basis of the level-of-spatial-ability approach. It was found that

Table 2

Proportions of CW, WW, CB, WB, and B B Items Over the Number of Non-CC Items on the Mental Rotations Test as a Function of Timing Condition (in Seconds) and Gender in Experiment 1

\begin{tabular}{|c|c|c|c|c|c|c|c|c|c|c|c|c|}
\hline \multirow{3}{*}{$\begin{array}{l}\text { Possible } \\
\text { Outcome } \\
\end{array}$} & \multicolumn{10}{|c|}{ Timing Condition } & & \\
\hline & \multicolumn{2}{|c|}{15} & \multicolumn{2}{|c|}{20} & \multicolumn{2}{|c|}{25} & \multicolumn{2}{|c|}{30} & \multicolumn{2}{|c|}{40} & \multicolumn{2}{|c|}{ Overall } \\
\hline & $M$ & $S D$ & $M$ & $S D$ & $M$ & $S D$ & $M$ & $S D$ & $M$ & $S D$ & $M$ & $S D$ \\
\hline \multicolumn{13}{|l|}{$\mathrm{CW}$} \\
\hline Women & 34.2 & 27.9 & 48.8 & 28.4 & 57.8 & 23.7 & 76.0 & 22.1 & 73.8 & 22.5 & 58.1 & 25.8 \\
\hline Men & 55.4 & 31.1 & 62.5 & 30.0 & 61.9 & 25.1 & 64.4 & 17.0 & 81.7 & 19.3 & 65.2 & 25.5 \\
\hline \multicolumn{13}{|l|}{ WW } \\
\hline Women & 6.4 & 8.4 & 8.9 & 10.4 & 11.5 & 10.8 & 9.8 & 9.8 & 9.4 & 10.3 & 9.2 & 10.7 \\
\hline Men & 10.9 & 11.0 & 8.1 & 8.6 & 11.7 & 11.1 & 14.2 & 14.6 & 7.1 & 11.2 & 10.4 & 10.6 \\
\hline \multicolumn{13}{|l|}{$\mathrm{CB}$} \\
\hline Women & 33.1 & 20.8 & 21.7 & 22.7 & 15.7 & 18.0 & 10.0 & 17.8 & 11.3 & 16.8 & 18.4 & 20.9 \\
\hline Men & 25.3 & 28.9 & 15.1 & 24.9 & 15.6 & 21.0 & 12.4 & 18.9 & 6.7 & 10.0 & 15.0 & 20.7 \\
\hline \multicolumn{13}{|l|}{ WB } \\
\hline Women & 10.8 & 10.3 & 8.8 & 12.7 & 5.9 & 8.1 & 2.5 & 5.8 & 1.8 & 4.1 & 6.0 & 8.4 \\
\hline Men & 5.2 & 7.4 & 4.0 & 6.6 & 3.8 & 5.8 & 4.1 & 7.3 & 4.1 & 8.9 & 4.2 & 8.3 \\
\hline \multicolumn{13}{|l|}{ BB } \\
\hline Women & 15.5 & 14.4 & 11.8 & 12.1 & 9.0 & 12.3 & 1.8 & 4.7 & 3.7 & 9.4 & 8.3 & 10.9 \\
\hline Men & 3.2 & 5.4 & 10.3 & 17.4 & 6.9 & 9.6 & 4.8 & 8.5 & 0.41 & 1.9 & 5.1 & 10.8 \\
\hline
\end{tabular}

Note-CW, one correct response and one incorrect response; WW, two incorrect responses; CB, one correct response and one blank; WB, one incorrect response and one blank; BB, two blanks. The proportions were calculated by dividing the number of $\mathrm{CW}, \mathrm{WW}, \mathrm{CB}, \mathrm{WB}$, or BB items by the number of non-CC items and reflect percentages. 
the average $\mathrm{CC}$ score for the males was higher than that for the females regardless of timing condition. Thus, on $\mathrm{CC}$ items, it appears that the gender difference is attributed to level of spatial ability rather than to performance factors, because gender differences of similar magnitude were found regardless of the time allotted on each item.

In addition to findings with direct relevance for a test of the performance factors interpretation, possible gender differences in items presumed to reflect guessing behavior were examined. In this case, support for Goldstein et al.'s (1990) hypothesis should reveal a smaller proportion of $\mathrm{CW}$ and $\mathrm{WW}$ items and a greater proportion of $\mathrm{CB}$, $\mathrm{WB}$, and $\mathrm{BB}$ items for the women than for the men. In contrast, findings of a greater proportion of $\mathrm{CW}$ and $\mathrm{WW}$ items and a smaller proportion of $\mathrm{CB}, \mathrm{WB}$, and $\mathrm{BB}$ items for the women than for the men would support Lohman's (1986) hypothesis. The proportion of CW and BB items showed a main effect of gender, suggesting that women have a lower propensity to guess than men, thus supporting the performance factors hypothesis.

The effect of timing conditions as well as the interaction between gender and timing provide elements of interpretation concerning the processes underlying test performance and how they might be affected by gender. The results showed an increase in the proportion of $\mathrm{CW}$ items as time available increases, whereas a decrease in the proportion of $\mathrm{CB}, \mathrm{WB}$, and $\mathrm{BB}$ items was found as time increases. This quite likely reflects the fact that, with more time available, the participants were less likely to leave a blank answer when they knew (from the instructions) that there are always two correct alternatives in each item. However, it does not mean that the answer was more readily available. This finding therefore suggests that the participants were more likely to guess when they had time to respond but did not know the correct answer-hence an increase in items reflecting guessing $(\mathrm{CW})$ and a decrease in items reflecting reluctance to guess $(\mathrm{CB}, \mathrm{WB}$, and $\mathrm{BB})$. Additionally, the gender $\times$ timing condition interaction generally reflected a smaller effect of timing in the men than in the women on items reflecting guessing. An examination of the pattern of performance on the relevant items in Table 2 suggests that women are likely to attempt a guess only if they are given enough time to produce what they consider a valid response (or an educated guess, so to speak), whereas men tend to guess regardless of whether enough time was available, which resulted in generally fewer items in which one of the responses was left blank. Essentially, the main effect of gender suggests that men emphasize more the need to produce two answers as stated in the instructions for the task, whereas women try to minimize their guessing, which constitutes another aspect of the instructions. However, the interaction between gender and timing suggests that women are more likely to guess than are men only when given enough time. The pattern of results across $\mathrm{CC}$ items and items reflecting guessing behavior suggests that the two hypotheses presented here are not necessarily mutually exclusive. In fact, performance factors likely interact with level of spatial ability to produce the pattern of results obtained in the present experiment. Specifically, women generally seem to work more carefully than men in terms of guessing behavior, but giving them more time does not allow them to reach the level of performance attained by men as reflected in number of $\mathrm{CC}$ items. However, before this conclusion is considered definite, it is important to review the limitations of the present experiment.

One such limitation concerns the fact that the time allotted for completion, even in the longest timing conditions in the present study, might have been too short to allow the participants to achieve an asymptotic level of performance on the CC items. It can also be argued plausibly that the mere fact that participants are given a finite amount of time to complete items is enough to create some sense of urgency, thus producing the results typically expected under stricter time constraints. The present findings are therefore still open to the argument that, with unlimited time, women would have been able to achieve a level of performance comparable with that of men. This suggests that an unlimited time condition might be required to reach that goal and to provide a clear test of the performance factors hypothesis. This would increase the probability that participants would achieve asymptotic performance. However, it is likely that such a condition would be better administered in an individual testing situation to avoid the influence of participants who complete the task at different speeds.

Another problematic aspect of the administration procedure used here is that the participants chose where they would sit in relation to the projection screen. This means that the stimuli appeared bigger to the participants sitting at the front of the room than to those sitting at the back. Although the stimuli were relatively large (see the Materials section) and the participants did not report upon debriefing that this factor affected their performance, it might have influenced the results. This would be especially true if the males and the females had clearly different seating preferences, and this factor was not examined here. It might be worthwhile to control this factor in future work by taking note of seating arrangements when testing occurs. Requiring participants to write down on the response sheet the row number of their seats would provide quantification of that information. This would allow that factor to be used in preliminary analyses to examine its possible influence on performance.

Finally, the participants were not randomly assigned to the different timing conditions, because they were tested during their class. This leaves open the argument that differences between conditions might be due to differences in group composition.

In view of these flaws, Experiment 2 was conducted to provide a more controlled test of the hypotheses. Specifically, in Experiment 2 extreme conditions (15 sec and unlimited time) as well as the duration corresponding to Voyer's (1997) allotted time (25 sec) were examined. The use 
Table 3

Mean Number of CC Items on the Mental Rotations Test and Percentage of Participants Attempting All Items as a Function of Timing Conditions (in Seconds) and Gender in Experiment 2

\begin{tabular}{|c|c|c|c|c|c|c|c|c|}
\hline \multirow[b]{3}{*}{ Group } & \multicolumn{6}{|c|}{ Timing Condition } & & \\
\hline & \multicolumn{2}{|c|}{15} & \multicolumn{2}{|c|}{25} & \multicolumn{2}{|c|}{ Unlimited } & \multicolumn{2}{|c|}{ Overall } \\
\hline & $M$ & $S D$ & $M$ & $S D$ & $M$ & $S D$ & $M$ & $S D$ \\
\hline \multicolumn{9}{|c|}{ CC Items } \\
\hline Women & 7.3 & 3.8 & 10.0 & 4.5 & 15.9 & 5.4 & 11.1 & 5.8 \\
\hline Men & 10.3 & 6.0 & 15.6 & 4.7 & 19.1 & 5.0 & 15.0 & 6.3 \\
\hline
\end{tabular}

Percentage of Participants Attempting All Items

\begin{tabular}{lllll} 
Women & 48.6 & 65.7 & 97.1 & 70.5 \\
Men & 42.9 & 68.6 & 94.3 & 68.6 \\
\hline
\end{tabular}

Note-CC, two correct responses. The percentage of participants attempting all items was computed within each gender.

of individual testing provided control over viewing distance and potential extraneous time pressure while allowing random assignment of the participants in the various conditions.

\section{EXPERIMENT 2}

\section{Method}

Participants. The participants were 105 female and 105 male undergraduate students enrolled in an introductory psychology course at the University of New Brunswick. The participants had normal or corrected-to-normal vision. Their participation was voluntary and was rewarded with course credit.

Materials and Procedure. The materials and procedure were similar to those of Experiment 1 except for the following changes. The participants were tested individually and were randomly assigned to one of three item duration conditions $(15 \mathrm{sec}, 25 \mathrm{sec}$, or unlimited time), with 35 participants of each gender in each condition. Test items were viewed directly on a computer screen positioned $50 \mathrm{~cm}$ away from the participants. In the $15-$ and $25-\mathrm{sec}$ conditions, the participants followed the same instructions as in Experiment 1 . In the untimed condition, the participants were instructed to press the space bar on the keyboard when they were ready to move on to the next trial.

In the presentation format used here, each of the five block configurations used on individual items was, on average, $1.9 \mathrm{~cm}$ wide and $1.9 \mathrm{~cm}$ high and was enclosed within a rectangular frame $2.6 \mathrm{~cm}$ high and $2.3 \mathrm{~cm}$ wide. The distance between the target stimulus and the first stimulus in each set of alternatives was $1.4 \mathrm{~cm}$, and a distance of $0.5 \mathrm{~cm}$ separated the alternatives.

\section{Results}

As in Experiment 1, the data were analyzed separately for each of the hypotheses. Therefore, an ANOVA with gender and timing condition ( $15 \mathrm{sec}, 25 \mathrm{sec}$, and unlimited duration) as the independent variables and the number of $\mathrm{CC}$ items as the dependent variable was computed to test the hypothesis concerning performance factors. A multivariate ANOVA with gender and timing condition as the independent variables and the proportion of $\mathrm{WW}, \mathrm{CB}$, $\mathrm{CW}, \mathrm{WB}$, and $\mathrm{BB}$ items as dependent variables was performed to test the hypothesis concerning guessing behavior. As was done in Experiment 1, Pillai's trace was used in the multivariate analyses of Experiment 2, and an alpha level of .05 was used in the power calculations.

CC as dependent variable. Analysis of the $\mathrm{CC}$ items revealed a significant effect of gender $[F(1,204)=32.79$, $\left.M S_{\mathrm{e}}=24.41, p<.01\right]$. Examination of the relevant means (see Table 3 ) reveals that the males obtained significantly more $\mathrm{CC}$ items than the females did.

A significant effect of timing condition was also found $\left[F(2,204)=53.67, M S_{\mathrm{e}}=24.41, p<.01\right]$. The means relevant to this finding are also presented in Table 3. Multiple comparisons with the Neuman-Keuls technique at the .05 level of significance revealed that differences in performance between all the conditions were significant, in the following order: unlimited $>25 \mathrm{sec}>15 \mathrm{sec}$.

The interaction between gender and timing condition failed to achieve significance $\left[F(2,204)=1.50, M S_{\mathrm{e}}=\right.$ 24.41 , power $=.32]$. The performance of men and that of women were therefore affected similarly by timing conditions.

Finally, the percentage of participants who attempted all items was examined (Table 3). No significant gender differences were observed in completion rate (smallest $p>$.40).

Proportions of $\mathrm{CB}, \mathrm{CW}, \mathrm{WB}, \mathrm{WW}$, and $\mathrm{BB}$ as dependent variables. Analysis of the proportion of $\mathrm{CB}, \mathrm{CW}$, $\mathrm{WB}, \mathrm{WW}$, and $\mathrm{BB}$ items revealed a significant multivariate effect of gender $[F(5,200)=4.21, p<.01]$. Univariate tests of significance indicated that the effect of gender was significant only on WB items $[F(1,204)=12.25$, $M S_{\mathrm{e}}=43.85, p<.01 ;$ minimum $p$ for other items $>.19$,

Table 4

Proportions of CW, WW, CB, WB, and BB Items Over the Number of Non-CC Items on the Mental Rotations Test as a Function of Timing Conditions (in Seconds) and Gender in Experiment 2

\begin{tabular}{|c|c|c|c|c|c|c|c|c|}
\hline \multirow{3}{*}{$\begin{array}{c}\text { Possible } \\
\text { Outcome }\end{array}$} & \multicolumn{6}{|c|}{ Timing Condition } & & \\
\hline & \multicolumn{2}{|c|}{15} & \multicolumn{2}{|c|}{25} & \multicolumn{2}{|c|}{ Unlimited } & \multicolumn{2}{|c|}{ Overall } \\
\hline & $M$ & $S D$ & $M$ & $S D$ & $M$ & $S D$ & $M$ & $S D$ \\
\hline \multicolumn{9}{|l|}{$\mathrm{CW}$} \\
\hline Women & 49.8 & 22.5 & 55.3 & 23.1 & 91.0 & 29.8 & 65.3 & 27.4 \\
\hline Men & 54.3 & 26.7 & 62.9 & 27.9 & 76.6 & 37.8 & 64.6 & 32.2 \\
\hline \multicolumn{9}{|l|}{ WW } \\
\hline Women & 11.3 & 11.3 & 10.6 & 10.3 & 6.1 & 9.6 & 9.3 & 10.6 \\
\hline Men & 9.1 & 10.4 & 6.2 & 9.9 & 6.2 & 18.3 & 7.2 & 13.4 \\
\hline \multicolumn{9}{|l|}{$\mathrm{CB}$} \\
\hline Women & 21.4 & 20.7 & 23.5 & 19.7 & 1.7 & 6.7 & 15.5 & 19.5 \\
\hline Men & 21.1 & 23.5 & 21.7 & 23.6 & 4.0 & 17.6 & 15.6 & 23.1 \\
\hline \multicolumn{9}{|l|}{ WB } \\
\hline Women & 10.7 & 9.9 & 6.5 & 7.2 & 1.0 & 3.3 & 6.0 & 8.3 \\
\hline Men & 4.3 & 6.5 & 3.2 & 6.4 & 1.0 & 4.3 & 2.8 & 5.9 \\
\hline \multicolumn{9}{|l|}{$\mathrm{BB}$} \\
\hline Women & 6.8 & 9.2 & 4.2 & 8.0 & 0.3 & 1.9 & 3.8 & 7.6 \\
\hline Men & 8.3 & 9.9 & 5.9 & 12.7 & 0.8 & 3.5 & 5.0 & 9.9 \\
\hline
\end{tabular}

Note- $\mathrm{CW}$, one correct response and one incorrect response; WW, two incorrect responses; $\mathrm{CB}$, one correct response and one blank; WB, one incorrect response and one blank; $\mathrm{BB}$, two blanks. The proportion was calculated by dividing the number of $\mathrm{CW}, \mathrm{WW}, \mathrm{CB}, \mathrm{WB}$, or $\mathrm{BB}$ items by the number of non-CC items and reflects a percentage. 
maximum power $=.25]$. An examination of the relevant means in Table 4 indicates that the women obtained a greater proportion of WB items than the men did.

A significant multivariate effect of timing condition was also found $[F(10,402)=8.84, p<.01]$. Univariate tests of significance indicated that the effect of timing condition was significant on all of the dependent variables except WW [CW: $F(2,204)=27.95, M S_{\mathrm{e}}=696.42, p<$ .01 ; CB: $F(2,204)=22.45, M S_{\mathrm{e}}=380.78, p<.01$; WB: $F(2,204)=16.95, M S_{\mathrm{e}}=43.85, p<.01 ; \mathrm{BB}: F(1,204)=$ $12.39, M S_{\mathrm{e}}=70.87, p<.01 ; \mathrm{WW}: F(1,204)=2.05$, $M S_{\mathrm{e}}=145.15$, n.s., power $\left.=.42\right]$. The means relevant to this finding are presented in Table 4. Multiple comparisons with the Neuman-Keuls technique at the .05 level of significance revealed that more $\mathrm{CW}$ items were obtained in the unlimited time condition than in the 25- and $15-\mathrm{sec}$ conditions. For WB, all the differences between the means of the conditions were significant $(5>25>$ unlimited). Finally, the unlimited time condition produced a significantly smaller proportion of CB and BB items than did the 15- and 25-sec conditions.

Finally, the gender $\times$ timing condition interaction was only marginally significant in the multivariate test $[F(10,402)=1.73, p<.072$, power $=.82]$. Considering the importance of this interaction for a test of the hypotheses, univariate tests of significance were examined despite the marginality of the multivariate test. The univariate tests indicated that this interaction was significant on $\mathrm{CW}\left[F(2,204)=3.55, M S_{\mathrm{e}}=696.42, p<.05\right]$ and $\mathrm{WB}\left[F(2,204)=4.19, M S_{\mathrm{e}}=43.85, p<.05\right.$; all other $p \mathrm{~s}>.55$, maximum power $=.15]$. The means relevant to this interaction are presented in Table 4. Simple main effect analyses on CW items revealed that the effect of timing condition was significant for both the females $\left[F(2,205)=25.25, M S_{\mathrm{e}}=693.15, p<.01\right]$ and the males $\left[F(2,205)=6.40, M S_{\mathrm{e}}=693.15, p<.05\right]$, whereas on WB items, timing condition was significant for the females $\left[F(2,205)=17.98, M S_{\mathrm{e}}=46.26, p<.01\right]$ but not for the males $\left[F(2,205)=2.06, M S_{\mathrm{e}}=46.26\right.$, n.s., power $\left.=.42\right]$. An examination of the magnitude of the $F$ tests and significance indicate that for both measures variations in time constraints affected the performance of the females more than that of the males.

\section{Discussion}

The purpose of Experiment 2 was to examine the same hypotheses that were investigated in Experiment 1, but with a better controlled procedure. Thus, $\mathrm{CC}$ items were expected to show a significant gender $\times$ timing condition interaction on the basis of the performance factors hypothesis, whereas gender differences of equal magnitude across timing conditions were expected on the basis of the level-of-spatial-ability approach. Gender differences in favor of the males on CC items did not interact with timing conditions, which supports an explanation in terms of the level-of-spatial-ability hypothesis rather than the performance factors hypothesis.
The examination of items presumed to reflect guessing behavior supported the notion that women have a lower propensity to guess than men, which supports the performance factors hypothesis. Specifically, the women generally obtained a greater proportion of WB items than the men did. The effect of timing conditions generally showed a greater proportion of $\mathrm{CW}$ items as time available increased, whereas a decrease in the proportion of $\mathrm{CB}, \mathrm{WB}$, and $\mathrm{BB}$ items was found as time increased. As in Experiment 1 , this finding is believed to indicate that the participants were more likely to guess when they had time to respond but did not know the correct answer. Although it stemmed from a marginal multivariate test of significance, the gender $\times$ timing condition interaction indicated a smaller effect of timing in the men than in the women on items reflecting guessing. On the basis of the rationale used to interpret the findings of Experiment 1, the results of Experiment 2 suggest that women tend to guess more when given enough time. In contrast, men have relatively stable guessing behavior, regardless of the time available for response production.

\section{GENERAL DISCUSSION}

In the present study, we examined two rival hypotheses accounting for performance on the MRT. Predictions based on the performance factors hypothesis, exemplified by Goldstein et al. (1990), were that gender differences on $\mathrm{CC}$ items would be reduced with relaxed time pressure. In contrast, the level-of-spatial-ability hypothesis, represented by Lohman (1986), predicted that gender differences would have a similar magnitude regardless of timing conditions. The results on the $\mathrm{CC}$ items are quite clear in supporting the level-of-spatial-ability hypothesis. Despite sufficient power, the gender $\times$ timing condition interaction failed to achieve statistical significance in both experiments. This finding is especially critical in Experiment 2 because, unlike Experiment 1, it included a condition in which the participants were given unlimited time to complete each trial. It is therefore legitimate to conclude from these findings that, even when given sufficient time, as a group women never achieve the level of performance attained by men on the MRT. It thus appears that they are limited by their level of spatial ability, as Lohman believed.

The analysis of CC items only provides a replication of previous works and, as such, does not reflect an original contribution to the literature. However, the examination of items presumed to reflect guessing behavior provides new insights into how men and women complete the MRT. Voyer (1997) had proposed that CW, WW, CB, and WB items reflect guessing and that a large number of these items would reflect a high propensity to guess. However, it was argued earlier that these items can be interpreted only when they are considered as a proportion of the number of non-CC items. In addition, items with one blank response (CB, WB) are more likely to reflect reluctance to guess than propensity to guess. Furthermore, because of 
the item-by-item administration used here, BB items lose their meaning as a measure of completion speed, especially when long exposure duration is used, and they should also be interpreted as reflecting reluctance to guess in the present context. In contrast, items in which two responses are produced but at least one incorrect choice is made $(\mathrm{CW}, \mathrm{WW})$ likely provide the most direct measure of guessing on the MRT. The use of these various measures as proportions of non-CC items within this interpretative framework thus provides a contribution with much potential for the interpretation of individual differences on the MRT.

In terms of the hypotheses considered here, Goldstein et al. (1990) claimed that women are less likely to guess. These authors would therefore predict that women should generally obtain a smaller proportion of $\mathrm{CW}$ and $\mathrm{WW}$ items than men do and a larger proportion of $\mathrm{CB}, \mathrm{WB}$, and $\mathrm{BB}$ items than men do. In contrast, the level-of-spatialability approach would support the position that women are more likely to guess than are men because they often cannot find the correct response, thus predicting the reverse pattern of gender differences than that predicted by the performance factors hypothesis. The results on non-CC items supported an interpretation based on Goldstein et al.'s performance factors hypothesis in both experiments.

The pattern of results obtained with measures reflecting guessing behavior is similar in the two experiments, although there are subtle differences. Both experiments showed a multivariate effect of gender (marginal in the multivariate test for Experiment 1), with the means suggesting more guessing by the men than by the women. However, this finding was significant on $\mathrm{CW}$ and $\mathrm{BB}$ in Experiment 1 and only on WB in Experiment 2. Although the data themselves do not provide a clear explanation of this difference, it is possible that factors other than the limitations of Experiment 1 might partly account for it. The procedure implemented here was designed to reduce the effect of group-related time pressure (e.g., when faster test takers fidget while they wait for the rest of the group to complete the test). However, in the longer timing conditions, some participants would complete the items quickly and would have time to behave in a way that makes this obvious. This would indirectly put pressure on slower participants to either complete items they cannot solve by guessing or leave them blank. This would result in more items that reflect this behavior in group administration, which suggests that the present implementation of the MRT might not fully control for implicit time pressures in group administration and that time pressures are likely more prominent in group administration than in individual administration.

Our findings related to the main effect of timing condition on guessing behavior are quite straightforward. Both experiments showed an effect of timing on all items except WW. In all cases, it is quite clear that as available time increases, the tendency to produce more items reflecting propensity to guess $(\mathrm{CW})$ and fewer items reflecting re- luctance to guess $(\mathrm{CB}, \mathrm{WB}$, and $\mathrm{BB})$ generally increases as well. To put it simply, this finding suggests that giving participants more time only ensures that they produce two responses per item, thus reducing the number of blank answers. In other words, knowledge of the correct responses is not necessarily affected by the amount of time available, and many items left blank when time is constrained are replaced by guesses at longer time intervals.

The effect of time available on guessing behavior tends to support a performance factors interpretation because it shows that the strategy one uses to complete the MRT depends on how much time is available for response. Specifically, guessing increases as time available increases. However, the gender $\times$ timing condition interaction (which is marginal for the multivariate test in Experiment 2) suggests a more subtle conclusion. In general, the guessing behavior of women seems to be affected more than that of men by the time available. This interaction implies that, although the present report and much of the relevant literature present the performance factors and level-of-spatialability hypotheses as mutually exclusive, these factors might actually combine to affect MRT performance. Specifically, men produce a relatively similar amount of guessing regardless of time limits. They are therefore applying a similar strategy under all conditions. As we stated in interpreting the findings of Experiment 1, men seem to be driven mostly by the need to produce two responses. In contrast, in women the amount of guessing increases when they have more time for item completion. This suggests that their approach to the task changes as they are given more time to respond. One possibility is that they become less careful in producing responses as the time they have increases. In fact, when time is short, women emphasize the part of the instructions concerning resistance to guessing. However, when more time is available, they seem to emphasize the aspect of the instructions requiring two answers for each item. Taken together, the findings indicate the influence of both the level of spatial ability, which limits the performance of both men and women as reflected by number of CC items, and the influence of performance factors, which affect the pattern of guessing as reflected by $\mathrm{CW}, \mathrm{CB}, \mathrm{WB}$, and $\mathrm{BB}$ items as a function of gender and time. This strongly suggests that level of spatial ability and performance factors jointly affect performance on the MRT.

A puzzling point arises when one considers how the present study fits into the literature concerning the influence of performance factors on the magnitude of gender differences on the MRT. The present study showed no change in the magnitude of gender differences on that test when time limits were relaxed, a finding similar to that reported by Resnick (1993) and Masters (1998). However, others have reported a reduction in the magnitude of gender differences when time limits are removed (Goldstein et al., 1990; Voyer, 1997). One is left wondering why such contradictory data might be found with the same test. However, when the studies are examined more closely, 
some findings can be set aside. Specifically, Experiment 1 of the present report differed from the experiments in the other studies in that it did not make use of an unlimited time condition. As was mentioned previously, Resnick used only an untimed condition, which precluded a test of the interaction that would reflect a reduction in the magnitude of the gender difference in the untimed condition. Finally, Voyer (1997) did not assign participants randomly to the time pressure conditions. It is therefore not possible to compare these three experiments with the remaining studies. Thus, we are left with three experiments: that of Goldstein et al. (1990), that of Masters, and Experiment 2 of the present study. Although Masters commented on the power issues relevant to Goldstein et al.'s experiments, one can still plausibly argue that gender differences were reduced with untimed administration. The remaining factor is that Goldstein et al. used a within-subjects manipulation of time constraints, whereas in the other two experiments a between-subjects design was used. This suggests that one could actually interpret Goldstein et al.'s data as reflecting a practice effect: Participants' performance was better on later items than on earlier ones, and so the magnitude of the gender difference was reduced. Of course, the possible influence of practice effects on Goldstein et al.'s findings is speculative, although Peters et al. (1995) did report that the MRT is highly susceptible to such effects. Nevertheless, it would require an item analysis to determine whether performance tends to be better on later items. It is worth noting that Stumpf (1998) presented this type of analysis on a 3-D mental rotation task called block rotation. Measures of performance were the item exposure time (IET) required for response on each individual item and accuracy of response. Although Stumpf did not report on the relation between item placement and accuracy, he found that the IET tended to decrease as participants progressed through the items. This supports the notion that practice effects took place. An item analysis of this sort has yet to be undertaken for the MRT. In addition to an investigation of possible practice effects, an item analysis of the MRT would allow an examination of accuracy as a function of the type of items. Specifically, the success rate might be higher for items for which the target and the distractors differ structurally as well as in orientation in comparison with those for which the only difference is in terms of orientation. However, Peters et al. reported that the magnitude of gender differences on their version of the MRT was not affected by changes in task difficulty. This suggests that an item analysis is unlikely to obtain variation in gender differences as a function of item type, although it remains an empirical question. For the moment, it appears that the contradictory findings present in the literature concerning well-controlled studies might be accounted for by the type of design used (i.e., between-subjects vs. within-subjects). The fact that Gallagher (1989) and Gallagher and Johnson (1992) obtained results similar to those of Goldstein et al. with the cube MRT (Johnson \& Meade, 1987) in a within-subjects design provides further support for this conclusion.
In conclusion, the present study replicated previous work demonstrating that the magnitude of gender differences on the MRT is not affected by the amount of time allotted for task completion. However, the critical contribution of the present study is in proposing a new way to analyze the various items observed on the MRT. Analysis of these items provided insight into how the time available affects guessing behavior. This analysis supported an interpretation by which performance factors and the level of spatial ability jointly affect gender differences on the MRT. The replication of most of the results of Experiment 1 in the better controlled Experiment 2 adds strength to this conclusion. The present study suggests that the gender difference on the MRT might in fact be due in part to performance factors. However, acceptance of the role of performance factors does not imply a rejection of the level-of-spatial-ability hypothesis, because the present data suggest that these factors are not mutually exclusive. Thus, the gender difference on the MRT is likely due to the joint effect of performance factors and level of spatial ability.

\section{REFERENCES}

Allen, M. J., \& Hogeland, R. (1978). Spatial problem-solving strategies as functions of sex. Perceptual \& Motor Skills, 47, 348-350.

AMERICAN PSYCHOLOGICAL AsSOCIATION (1992). Ethical principles of psychologists and code of conduct. American Psychologist, 47, 1597 1611.

BAenninger, M., \& Newcombe, N. (1989). The role of experience in spatial test performance: A meta-analysis. Sex Roles, 20, 327-344.

CAPlan, P. J., MacPherson, G. M., \& Tobin, P. (1985). Do sex-related differences in spatial abilities exist? A multilevel critique with new data. American Psychologist, 40, 786-799.

Gallagher, S. A. (1989). Predictors of SAT mathematics scores of gifted male and gifted female adolescents. Psychology of Women Quarterly, 13, 191-203.

Gallagher, S. A., \& Johnson, E. S. (1992). The effect of time limits on performance of mental rotations by gifted adolescents. Gifted Child Quarterly, 36, 19-22.

Goldstein, D., Haldane, D., \& Mitchell, C. (1990). Sex differences in visual-spatial ability: The role of performance factors. Memory \& Cognition, 18, 546-550.

Johnson, E., \& MEADE, A. (1987). Developmental patterns of spatial ability: An early sex difference. Child Development, 58, 725-740.

LEVY, J. (1971). Lateral specialization of the human brain: Behavioral manifestations and possible evolutionary basis. In J. A. Kiger, Jr. (Ed.), The biology of behavior (pp. 159-180). Corvallis: Oregon State University Press.

Linn, M. C., \& Petersen, A. C. (1985). Emergence and characterization of sex differences in spatial ability: A meta-analysis. Child Development, 56, 1479-1498.

LOHMAN, D. F. (1986). The effect of speed-accuracy tradeoff on sex differences in mental rotation. Perception \& Psychophysics, 39, 427-436.

MASTERS, M. S. (1998). The gender difference on the Mental Rotations Test is not due to performance factors. Memory \& Cognition, 26, 444448.

McGee, M. G. (1979). Human spatial abilities: Psychometric studies and environmental, genetic, hormonal, and neurological influences. Psychological Bulletin, 86, 889-911.

Peters, M., Laeng, B., Latham, K., Johnson, M., Zaiyouna, R., \& Richardson, C. (1995). A redrawn Vandenberg and Kuse Mental Rotations Test: Different versions and factors that affect performance. Brain \& Cognition, 28, 39-58.

RESNICK, S. M. (1993). Sex differences in mental rotations: An effect of time limits? Brain \& Cognition, 21, 71-79. 
SAnders, B., \& SoAres, M. P. (1986). Sexual maturation and spatial ability in college students. Developmental Psychology, 22, 199-203.

ShePARD, R. N., \& MetZler, J. (1971). Mental rotation of threedimensional objects. Science, 171, 701-703.

SignORELLA, M. L., \& JAMISON, W. (1986). Masculinity, femininity, androgyny, and cognitive performance: A meta-analysis. Psychological Bulletin, 100, 207-228.

STUMPF, H. (1998). Gender-related differences in academically talented students' scores and use of time on tests of spatial ability. Gifted Child Quarterly, 42, 157-171.

TABACHNICK, B. G., \& FIDELL, L. S. (1996). Using multivariate statistics (3rd ed.). New York: Harper Collins.

Vandenberg, S. G. (1985). Answer Key for Mental Rotations Test. Boulder: Author.

Vandenberg, S. G., \& Kuse, A. R. (1978). Mental Rotation, a group test of three-dimensional spatial visualization. Perceptual \& Motor Skills, 47, 599-604.

VOYER, D. (1997). Scoring procedure, performance factors, and magnitude of sex differences in spatial performance. American Journal of Psychology, 110, 259-276.
Voyer, D., Voyer, S., \& Bryden, M. P. (1995). Magnitude of gender differences in spatial abilities: A meta-analysis and consideration of critical variables. Psychological Bulletin, 117, 250-271.

WABER, D. P. (1976). Sex differences in cognition: A function of maturation rate? Science, 192, 572-574.

\section{NOTE}

1. In the pilot, the present task was implemented with an exposure duration of $10 \mathrm{sec}$ in a sample of 24 participants ( 16 males, 8 females). The results showed a clear floor effect, with the number of $\mathrm{CB}$ items $(M=$ $5.46, S D=4.28)$ and $C W$ items $(M=7.83, S D=4.24)$ exceeding the number of $C C$ items $(M=4.62, S D=2.45)$ and no gender differences on any of the measures.

(Manuscript received May 30, 2002;

revision accepted for publication August 15, 2003.) 